\title{
List of Illustrations Liste des illustrations
}

Fig. 1.1

Contact sheet 50-3-9, negative no. 6

Planche contact 50-3-9, négatif $n^{\circ} 6$

Fig. 1.2

Contact sheet $50-3-10$, negative no. 21 (picture is inverted)

Planche contact 50-3-10, négatif $n^{\circ} 21$ (la photo est inversée)

Fig. 1.3

Contact sheet 50-3-11, negative no. 31

Planche contact 50-3-11, négatif $n^{\circ} 31$

Fig. 1.4

Contact sheet 50-3-13, negative no. 53

Planche contact 50-3-13, négatif $\mathrm{n}^{\circ} 53$

Fig. 1.5

Contact sheet 50-3-13, negative no. 59

Planche contact 50-3-13, négatif $n^{\circ} 59$

Fig. 1.6

Contact sheet 50-3-14, negative no. 69

Planche contact 50-3-14, négatif $n^{\circ} 69$

\section{Fig. 1.7}

Contact sheet 50-3-16, negative no. 92

Planche contact 50-3-16, négatif $n^{\circ} 92$

Fig. 1.8

Contact sheet 50-3-18, negative no. 117

Planche contact 50-3-18, négatif $\mathrm{n}^{\mathrm{O}} 117$ 
Fig. 1.9

Contact sheet 50-3-19, negative no. 127 Planche contact 50-3-19, négatif $n^{\circ} 127$

Fig. 1.10

Contact sheet 50-3-20, negative no. 139 Planche contact 50-3-20, négatif $n^{\circ} 139$

Fig. 1.11

Contact sheet 50-3-21, negative no. 152 Planche contact 50-3-21, négatif $n^{\circ} 152$

Fig. 1.12

Contact sheet 50-3-24, negative no. 190 Planche contact 50-3-24, négatif $n^{\circ} 190$

Fig. 1.13

Contact sheet 50-3-23, negative no. 172 Planche contact 50-3-23, négatif $\mathrm{n}^{\mathrm{o}} 172$

\section{Fig. 1.14}

Contact sheet 50-3-25, negative no. 197 Planche contact 50-3-25, négatif n ${ }^{\circ} 197$

\section{Fig. 1.15}

Contact sheet 50-3-25, negative no. 199 Planche contact 50-3-25, négatif n ${ }^{\circ} 199$

\section{Fig. 1.16}

Contact sheet 50-3-26, negative no. 207 Planche contact 50-3-26, négatif n 207

\section{Fig. 1.17}

Contact sheet 50-3-26, negative no. 206 Planche contact 50-3-26, négatif $n^{\circ} 206$ 


\section{Fig. 1.18}

Contact sheet 50-3-28, negative no. 231

Planche contact 50-3-28, négatif $n^{\circ} 231$

Fig. 1.19

Contact sheet 50-3-27, negative no. 220

Planche contact 50-3-27, négatif $n^{\circ} 220$

Fig. 1.20

Contact sheet 50-3-30, negative no. 254 (picture is inverted)

Planche contact 50-3-30, négatif $n^{\circ} 254$ (la photo est inversée)

Fig. 1.21

Contact sheet 50-3-29, negative no. 250

Planche contact 50-3-29, négatif $n^{\circ} 250$

Fig. 1.22

Contact sheet 50-3-32, negative no. 286 (picture is inverted)

Planche contact 50-3-32, négatif $n^{\circ} 286$ (la photo est inversée)

Fig. 1.23

Contact sheet 50-3-32, negative no. 277

Planche contact 50-3-32, négatif $n^{\circ} 277$

Fig. 1.24

Contact sheet 50-3-33, negative no. 289

Planche contact 50-3-33, négatif n ${ }^{\circ} 289$

\section{Fig. 1.25}

Contact sheet 50-3-34, negative no. 324 (picture is inverted)

Planche contact 50-3-34, négatif $n^{\circ} 324$ (la photo est inversée)

\section{Fig. 1.26}

Contact sheet 50-3-34, negative no. 313 (picture is inverted)

Planche contact 50-3-34, négatif $n^{\circ} 313$ (la photo est inversée) 
Fig. 1.27

Contact sheet 50-3-35, negative no. 330

Planche contact 50-3-35, négatif $n^{\circ} 330$

Fig. 1.28

Contact sheet 50-3-36, negative no. 347

Planche contact 50-3-36, négatif n ${ }^{\circ} 347$

Fig. 1.29

Contact sheet 50-3-38, negative no. 309

Planche contact 50-3-38, négatif $n^{0} 309$

Fig. 1.30

Leica 1, negative no. 29

Leica 1 , négatif $n^{\circ} 29$ 